\title{
Quarkonium Spectral Function from Anisotropic Lattice
}

\author{
Alexander Velytsky \\ Department of Physics and Astronomy, UCLA, Los Angeles, CA 90095-1547, \\ $U S A$
}

\begin{abstract}
We discuss the behavior of charmonia and bottomonia correlators and spectral functions above the deconfinement temperature and determine melting temperatures for different mesonic states.
\end{abstract}

Key words: lattice QCD, heavy quarkonium, spectroscopy

PACS: 11.15.Ha, 11.10.Wx, 12.38.Mh, 25.75.Nq

\section{Introduction}

The study of heavy quarkonia at finite temperature is an important way to probe hot and dense strongly interacting matter. General considerations suggest that quarkonia will melt at temperatures somewhat higher than the deconfinement temperature as a result of modification of interquark potential due to color screening. Matsui and Satz [1] proposed that color screening at high temperature will lead to quarkonium suppression, which can be used as a signal of QGP formation. Studies of modification of quarkonium potential in terms of spectral functions have started to appear only recently $[2,3,4,5]$. However, the systematic errors were not well controlled and it is not clear which features of the charmonium spectral functions are physical and which are lattice artifacts of the MEM analysis and/or finite lattice spacing.

This contribution reports updated results of our study of charmonium $[6,7]$ and bottomonium [8] spectral functions and correlators at finite temperature. The calculations are performed in quenched QCD using anisotropic lattices, the Wilson gauge action and the Fermilab fermion action [11]. Further details of the lattice action and the parameters of the simulations can be found in [6]. Detailed results of the study and description of the Maximum Entropy Method (MEM) will be presented in our upcoming paper [9]. 


\section{Meson Correlators and Spectral Functions}

We look at correlators of point meson operator $J_{H}(t, x)=\bar{q}(t, x) \Gamma_{H} q(t, x)$, where $\Gamma_{H}=1, \gamma_{5}, \gamma_{\mu}, \gamma_{5} \gamma_{\mu}, \gamma_{\mu} \gamma_{\nu}$ fixes the mesonic channel to (pseudo)scalar, (pseudo)vector and tensor channels. On the lattice one computes the Euclidean propagator $G_{H}(\tau, \vec{p})=\int d^{3} x e^{i \vec{p} \cdot \vec{x}}\left\langle T_{\tau} J_{H}(\tau, \vec{x}) J_{H}(0, \overrightarrow{0})\right\rangle$, which is related to the spectral function through the integral representation

$$
G(\tau, \vec{p})=\int_{0}^{\infty} d \omega \sigma K(\omega, \tau), \quad K(\omega, \tau)=\frac{\cosh (\omega(\tau-1 / 2 T))}{\sinh (\omega / 2 T)}
$$

Spectral functions can be reconstructed using Bayesian techniques, such as the MEM [10]. Instead of using Bryan's algorithm we use a new algorithm, which will be described in details in [9].

First we check the MEM ability to reconstruct a model spectral function of finite width. For a model spectral function we choose the Breit-Wigner model spectral function, which (in physical units) is given by

$$
\sigma(\omega)=r_{1} \frac{1}{\pi} \frac{\gamma}{(\omega-m)^{2}+\gamma^{2}} \frac{\omega^{3}}{m^{3}} * \frac{3}{2 \pi}
$$

The covariance matrix from an actual lattice simulation of charmonium at zero temperature $\left(\beta=6.5, \xi=4, N_{t}=160\right)$ is used to introduce the noise. In Fig. 1 we show the model and reconstructed (with $m(\omega)=0.038 \omega^{2}$ and $m(\omega)=1$ default models) spectral functions for the pseudoscalar channel. We see that the MEM can successfully reconstruct the model spectral function. However, it is gradually loosing its ability as we increase the width and at $\gamma=2 \mathrm{GeV}$ is incapable of spectral function reconstruction.
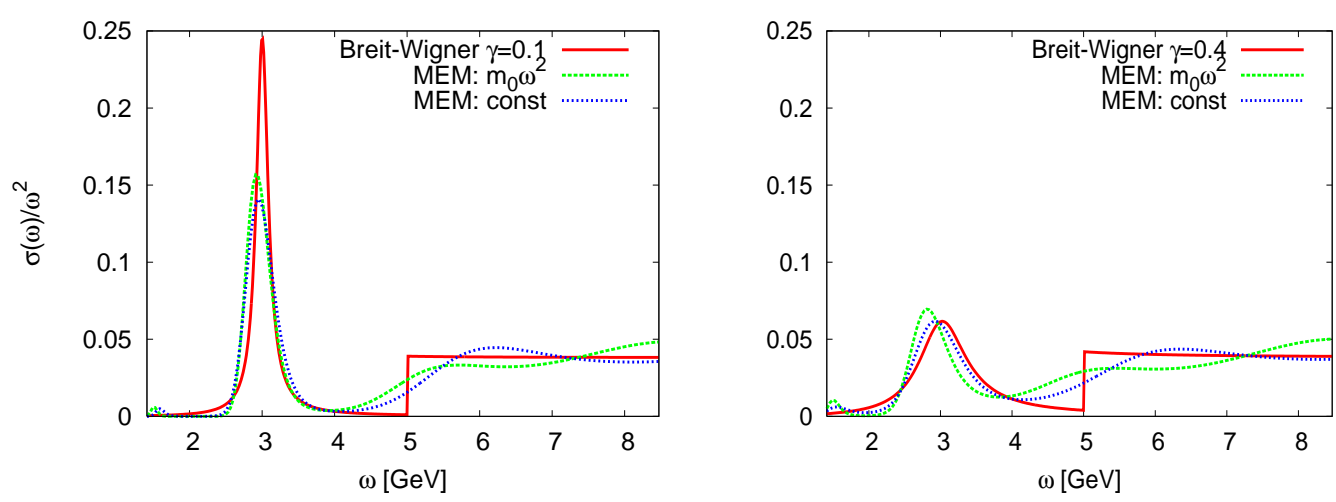

Fig. 1. Reconstruction of the $\gamma=0.1$ and $0.4 \mathrm{GeV}$ Breit-Wigner model spectral functions. 


\section{Spectral functions and correlators at finite temperature}

With increasing temperature it becomes more and more difficult to reconstruct the spectral functions, therefore it is useful to study the temperature dependence of quarkonia correlators first. To separate out the trivial temperature dependence due to the integration kernel, following Ref. [5] at each temperature we calculate the so-called reconstructed correlator

$$
G_{\text {recon }}(\tau, T)=\int_{0}^{\infty} d \omega \sigma(\omega, T=0) K(\tau, \omega, T)
$$

The ratio of the original and the reconstructed correlator should be close to one, $G(\tau, T) / G_{\text {recon }}(\tau, T) \sim 1$, if there is no temperature dependence in the spectral function. In Fig. 2 we show the ratio of the correlators for charmonium in pseudoscalar and scalar channels. It is evident that there is little modification of the correlator in the pseodoscalar channel for temperatures up to $1.5 T_{c}$. For the scalar channel, however, the change in the correlator is noticeable at temperatures as low as $1.16 T_{c}$. The results for $\beta=6.1$ and 6.5 are very similar, thus there is little dependence on the lattice spacing. In Fig.
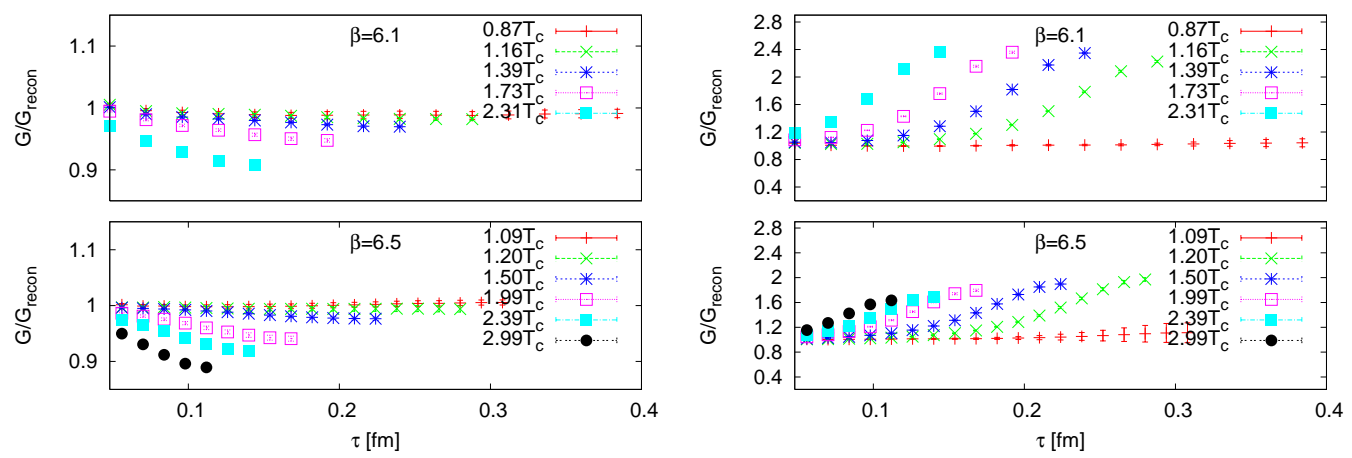

Fig. 2. The ratio $G(\tau, T) / G_{\text {recon }}(\tau, T)$ of charmonium for pseudoscalar (left) and scalar (right) channels at $a_{t}^{-2}=8.18$ and $14.11 \mathrm{GeV}$ at different temperatures.

3 we present charmonium and bottomonium spectral functions for the scalar channel at different temperatures. From these figures the dissolution of the ground states at temperatures above deconfinement is clear. For charmonium we tried two different default models $m(\omega)=0.038 \omega^{2}$ and $m(\omega)=1$ and the results are qualitatively the same, which is another check on systematics.

\section{Summary}

From our studies of correlators and spectral functions we can conclude that, the $1 S\left(\eta_{c}, J / \psi\right)$ charmonium states exist as a resonance in the deconfined 

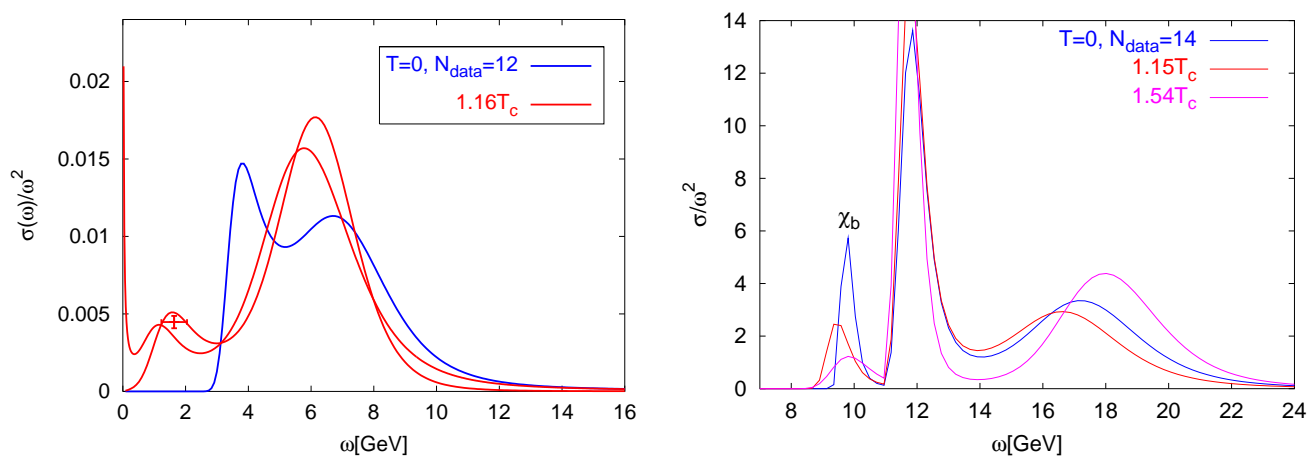

Fig. 3. Charmonia (left) and bottomonia (right) spectral functions in scalar channel for different temperatures.

phase at $T \simeq 1.5 T_{c}$. On the other hand, the $1 P\left(\chi_{c 0}, \chi_{c 1}\right)$ charmonium states dissolve at $1.1 T_{c}$. Bottomonium states show similar behavior.

\section{Acknowledgments}

This presentation is done based on work in collaboration with A. Jakovác, P. Petreczky, and K. Petrov. This work is partially supported by NSF-PHY0309362 .

\section{References}

[1] T. Matsui and H. Satz, Phys. Lett. B 178, 416 (1986).

[2] S. Datta et al, Nucl. Phys. Proc. Suppl. 119, 487 (2003) arXiv:hep-lat/0208012.

[3] T. Umeda, K. Nomura and H. Matsufuru, arXiv:hep-lat/0211003.

[4] M. Asakawa and T. Hatsuda, Phys. Rev. Lett. 92, 012001 (2004) arXiv:hep-lat/0308034.

[5] S. Datta et al, Phys. Rev. D 69, 094507 (2004) arXiv:hep-lat/0312037.

[6] A. Jakovác, P. Petreczky, K. Petrov and A. Velytsky, arXiv:hep-lat/0603005.

[7] P. Petreczky et al, PoS LAT2005, 185 (2006) arXiv:hep-lat/0510021.

[8] K. Petrov et al, PoS LAT2005, 153 (2006) arXiv:hep-lat/0509138.

[9] A. Jakovác, P. Petreczky, K. Petrov and A. Velytsky, work in progress.

[10] M. Asakawa et al, Prog. Part. Nucl. Phys. 46, 459 (2001) arXiv:hep-lat/0011040.

[11] A. X. El-Khadra et al, Phys. Rev. D 55, 3933 (1997) arXiv:hep-lat/9604004. 